\title{
SECOND ORDER LINEAR AND NONLINEAR DIFFERENTIAL EQUATIONS'
}

\author{
J. J. GERGEN AND F. G. DRESSEL
}

1. Introduction. It was noted by Pinney [2] that the solution of the nonlinear differential equation $y^{\prime \prime}+p(x) y^{\prime}+c y^{-3}=0, c$ constant, can be written in the form $y=\left(u_{1}^{2}-u_{2}^{2}\right)^{1 / 2}$, where $u_{1}(x), u_{2}(x)$ are appropriately chosen solutions of the linear equation $u^{\prime \prime}+p(x) u=0$. This result led Thomas [3] to ask: What equations of order $n$ have general solutions expressible in the form $y=F\left(u_{1}, \cdots, u_{n}\right)$, where $u_{1}, \cdots, u_{n}$ constitute a variable set of solutions of a linear equation? Thomas answered this question when the underlying linear equation is of the first order, $u^{\prime}+p u=q$. He also gave the answer for homogeneous second order equations, $u^{\prime \prime}+p u^{\prime}+q u=0$, when $F$ depends only on one $u$, or when $F$ is homogeneous of nonzero degree in two $u$ 's. Using the theory of passive partial differential equations, Herbst [1] removed these restrictions, obtaining the following general theorem.

THEOREM 1. If $u_{1}(x), u_{2}(x)$ are variable independent solutions with Wronskian w of the linear equation

$$
u^{\prime \prime}=w^{\prime} w^{-1} u^{\prime}+q u,
$$

where $w(x), q(x)$ are arbitrarily prescribed functions, then the equation

$$
y^{\prime \prime}=w^{\prime} w^{-1} y^{\prime}+f\left(y, y^{\prime}, w, q\right)
$$

has general solution $y=F\left(u_{1}, u_{2}\right)$ if, and only if, $f$ has the form

$$
f=q Z(y)+A(y)\left(y^{\prime}\right)^{2}+C(y) w^{2},
$$

where $Z, A, C$ satisfy

$$
Z^{\prime}-A Z=1, \quad Z C^{\prime}+(3-A Z) C=0 .
$$

Herbst's theorem determines the form of $f$. In Herbst's analysis, however, $F$ is determined only as a solution of a system of four partial differential equations. The purpose of this paper is to give a simple characterization of $F$. On the basis of information obtained a method is developed for the solution of (1.2) when $f$ has form (1.3) and $Z, A, C$ satisfy (1.4).

Received by the editors April 23, 1964.

1 This research was supported by the United States Air Force under grants AFOSR 61-51 and 62-162 monitored by the Air Force Office of Scientific Research. 
2. Main result. For the determination of $F$ only a limited class of linear equations (1.1) is needed, namely, the class for which $w$ and $q$ are constants, $w \neq 0$. Our main result is as follows.

Theorem 2. Suppose that $f\left(y, y^{\prime}, w, q\right) \in C^{\prime}$ on a domain $R=$ $\left\{\left(y, y^{\prime}, w, q\right) \mid m<y<M\right\}$, that

$$
\xi(y) \equiv f(y, 0,0,1) \neq 0, \quad m<y<M,
$$

and that $F\left(u_{1}, u_{2}\right) \in C^{\prime \prime}$ and $m<F<M$ on a domain $V$. Suppose further that for arbitrary constants $w \neq 0$ and $q$, if $u_{1}(x), u_{2}(x)$ are solutions of (1.1) with Wronskian w such that $\left(u_{1}, u_{2}\right) \in V$ for $x$ on an interval $I$, then $y=F\left(u_{1}, u_{2}\right)$ satisfies (1.2) on I. Under these conditions, if $m<\eta$ $<M$, then $F$ can be written

$$
F=\Phi\left(\omega^{1 / 2}\right), \quad\left(u_{1}, u_{2}\right) \in V,
$$

where $\omega\left(u_{1}, u_{2}\right)$ is a homogeneous polynomial of degree 2 , positive in $V$, and $\Phi$ is the inverse of

$$
\phi(y)=\exp \left\{\int_{\eta}^{y} \xi^{-1}(t) d t\right\}, \quad m<y<M .
$$

The proof is based on the uniqueness property of solutions of differential equations. Knowledge of the form (1.3) of $f$ is not required. We observe that in the Pinney case $\omega=u_{1}^{2}-u_{2}^{2}$ and $\Phi(y)=y$.

Proof. We show first that

$$
L\left(u_{1}, u_{2}\right) \equiv u_{1} F_{1}\left(u_{1}, u_{2}\right)+u_{2} F_{2}\left(u_{1}, u_{2}\right)=\xi\left(F\left(u_{1}, u_{2}\right)\right),\left(u_{1}, u_{2}\right) \in V
$$

where $F_{i}=\partial F / \partial u_{i}, i=1,2$. Let $\left(v_{1}, v_{2}\right)$ be a point of $V$ other than the origin. Let $v_{1}^{\prime}, v_{2}^{\prime}$ be arbitrarily chosen so that $w=v_{1} v_{2}^{\prime}-v_{2} v_{1}^{\prime} \neq 0$. Let $u_{1}(x), u_{2}(x)$ be the solution on $(-\infty, \infty)$ of $u^{\prime \prime}=u$ with initial values $u_{i}(0)=v_{i}, u_{i}^{\prime}(0)=v_{i}^{\prime}$. Then $u_{1}, u_{2}$ have Wronskian $w$, and $\left(u_{1}, u_{2}\right) \in V$ for $x$ on an interval $I$ containing $x=0$. Hence, by hypothesis, $y=F\left(u_{1}, u_{2}\right)$ satisfies $y^{\prime \prime}=f\left(y, y^{\prime}, w, 1\right)$ on $I$. Differentiating $y$ twice, and placing $x=0$ in the final equation, we get

$$
\begin{aligned}
v_{1} F_{1}+v_{2} F_{2}+F_{11}\left(v_{1}^{\prime}\right)^{2}+2 F_{12} v_{1}^{\prime} v_{2}^{\prime} & +F_{22}\left(v_{2}^{\prime}\right)^{2} \\
& =f\left(F, v_{1}^{\prime} F_{1}+v_{2}^{\prime} F_{2}, w, 1\right),
\end{aligned}
$$

where $F, F_{i}, F_{i j}$ are evaluated at $\left(v_{1}, v_{2}\right)$. With $v_{1}, v_{2}$ fixed we can let $v_{1}^{\prime}, v_{2}^{\prime} \rightarrow 0$ in $(2.5)$, to obtain $L\left(v_{1}, v_{2}\right)=f\left(F\left(v_{1}, v_{2}\right), 0,0,1\right)$. Hence, (2.4) holds save possibly at the origin. We observe, however, that the origin cannot be a point of $V$. Otherwise, by continuity, we would have $\xi(F(0,0))=L(0,0)=0$, contrary to $(2.1)$.

Now let $P_{0}:\left(v_{1}^{0}, v_{2}^{0}\right)$ be a fixed point in $V$. Let $V_{0}$ be an open disk in 
$V$ having center at $P_{0}$. We prove that there exist constants $a_{0}, b_{0}, c_{0}$, not all zero, such that

$$
\left(b_{0} u_{1}+c_{0} u_{2}\right) F_{1}\left(u_{1}, u_{2}\right)-\left(a_{0} u_{1}+b_{0} u_{2}\right) F_{2}\left(u_{1}, u_{2}\right)=0, \quad\left(u_{1}, u_{2}\right) \in V_{0} .
$$

By (2.1) and (2.4), the gradient of $F$ is different from zero at $P_{0}$. Accordingly, there are functions $\lambda_{i}(s), i=1,2$, of class $C^{\prime \prime}$ on an interval $|s|<\sigma$, where $0<\sigma$, such that $\lambda_{i}(0)=v_{i}^{0}, 0<\left(\lambda_{1}^{\prime}(0)\right)^{2}+\left(\lambda_{2}^{\prime}(0)\right)^{2}$, and

$$
\left(\lambda_{1}, \lambda_{2}\right) \in V_{0}, \quad F\left(\lambda_{1}, \lambda_{2}\right)=F\left(v_{1}^{0}, v_{2}^{0}\right), \quad|s|<\sigma .
$$

Let $\left(v_{1}, v_{2}\right)$ be a second point of $V_{0}$ such that $w=v_{1}^{0} v_{2}-v_{2}^{0} v_{1} \neq 0$. Since $L\left(\lambda_{1}(s), \lambda_{2}(s)\right) \neq 0$, the equation

$$
\left(\begin{array}{cc}
F_{1}\left(\lambda_{1}, \lambda_{2}\right) & F_{2}\left(\lambda_{1}, \lambda_{2}\right) \\
-\lambda_{2} & \lambda_{1}
\end{array}\right)\left(\begin{array}{c}
z_{1} \\
z_{2}
\end{array}\right)=\left(\begin{array}{cc}
F_{1}\left(v_{1}^{0}, v_{2}^{0}\right) & F_{2}\left(v_{1}^{0}, v_{2}^{0}\right) \\
0 & v_{2}^{0} \\
-v_{2} & v_{1}
\end{array}\right)\left(\begin{array}{l}
v_{1} \\
v_{2}
\end{array}\right)
$$

admits for each $s$ on $(-\sigma, \sigma)$, a unique solution $z_{i}=z_{i}(s)$. From (2.4) and (2.7) we have

$$
L\left(\lambda_{1}, \lambda_{2}\right)=L\left(v_{1}^{0}, v_{2}^{0}\right), \quad|s|<\sigma .
$$

Hence,

$$
\left(\begin{array}{l}
z_{1}(s) \\
z_{2}(s)
\end{array}\right)=\left(\begin{array}{ll}
\alpha(s) & \beta(s) \\
\gamma(s) & \delta(s)
\end{array}\right)\left(\begin{array}{l}
v_{1} \\
v_{2}
\end{array}\right)
$$

where

$$
L\left(v_{1}^{0}, v_{2}^{0}\right)\left(\begin{array}{ll}
\alpha & \beta \\
\gamma & \delta
\end{array}\right)=\left(\begin{array}{cc}
\lambda_{1} & -F_{2}\left(\lambda_{1}, \lambda_{2}\right) \\
\lambda_{2} & F_{1}\left(\lambda_{1}, \lambda_{2}\right)
\end{array}\right)\left(\begin{array}{cc}
F_{1}\left(v_{1}^{0}, v_{2}^{0}\right) & F_{2}\left(v_{1}^{0}, v_{2}^{0}\right) \\
-v_{2}^{0} & v_{1}^{0}
\end{array}\right)
$$

We note that $z_{i}=v_{i}$ for $s=0$. Hence, for $|s|$ sufficiently small, $|s|<\sigma_{1}$, say, where $0<\sigma_{1} \leqq \sigma$, we have $\left(z_{1}(s), z_{2}(s)\right) \in V_{0}$.

Suppose for the moment $s$ held fast on $\left(-\sigma_{1}, \sigma_{1}\right)$, and consider the linear functions $u_{i}(x)=u_{i}(x, s)=(1-x) \lambda_{i}+x z_{i}, 0 \leqq x \leqq 1$. We have $u_{i}(0)=\lambda_{i}, u_{i}(1)=z_{i}$. Since $V_{0}$ is convex it follows that $\left(u_{1}, u_{2}\right) \in V_{0}$, $0 \leqq x \leqq 1$. Further, $u_{1}, u_{2}$ satisfy $u^{\prime \prime}=0$ and, by (2.8), have Wronskian

$$
\lambda_{1}\left(z_{2}-\lambda_{2}\right)-\lambda_{2}\left(z_{1}-\lambda_{1}\right)=\lambda_{1} z_{2}-\lambda_{2} z_{1}=\stackrel{0}{v_{1} v_{2}}-\stackrel{0}{v_{2} v_{1}} \text {. }
$$

Hence, $y=y(x)=y(x, s)=F\left(u_{1}(x, s), u_{2}(x, s)\right)$ satisfies

$$
y^{\prime \prime}=f\left(y, y^{\prime}, w, 0\right), \quad 0 \leqq x \leqq 1 .
$$

The equation (2.12) is independent of $s$. Referring to (2.7), (2.8), and (2.9), we verify that 


$$
\begin{aligned}
y(0, s) & =F\left(\lambda_{1}(s), \lambda_{2}(s)\right)=F\left(v_{1}^{0}, v_{2}^{0}\right) \\
y^{\prime}(0, s) & =\left(z_{1}-\lambda_{1}\right) F_{1}\left(\lambda_{1}, \lambda_{2}\right)+\left(z_{2}-\lambda_{2}\right) F_{2}\left(\lambda_{1}, \lambda_{2}\right) \\
& =v_{1} F_{1}\left(v_{1}^{0}, v_{2}^{0}\right)+v_{2} F_{2}\left(v_{1}^{0}, v_{2}^{0}\right)-L\left(v_{1}^{0}, v_{2}^{0}\right) .
\end{aligned}
$$

Thus, $y(x, s), y^{\prime}(x, s)$ have the same initial values, $y(0, s), y^{\prime}(0, s)$, independently of $s$. Since $f \in C^{\prime}$ on $R$, we conclude from the uniqueness property of solutions of differential equations that $y(x, s)$ is independent of $s$ on $|s|<\sigma_{1}$. Taking $x=1$ we obtain

$$
F\left(z_{1}(s), z_{2}(s)\right)=F\left(z_{1}(0), z_{2}(0)\right)=F\left(v_{1}, v_{2}\right), \quad|s|<\sigma_{1} .
$$

We now differentiate in (2.14) and place $s=0$. Using (2.10) we get

$$
\left(\alpha^{\prime}(0) v_{1}+\beta^{\prime}(0) v_{2}\right) F_{1}\left(v_{1}, v_{2}\right)+\left(\gamma^{\prime}(0) v_{1}+\delta^{\prime}(0) v_{2}\right) F_{2}\left(v_{1}, v_{2}\right)=0 .
$$

From (2.11) we obtain

$$
\begin{aligned}
& L \alpha^{\prime}(0)=\lambda_{1}^{\prime} F_{1}+v_{2}^{0}\left(\lambda_{1}^{\prime} F_{12}+\lambda_{2}^{\prime} F_{22}\right), \\
& L \beta^{\prime}(0)=\lambda_{1}^{\prime} F_{2}-v_{1}^{0}\left(\lambda_{1}^{\prime} F_{12}+\lambda_{2}^{\prime} F_{22}\right), \\
& L \gamma^{\prime}(0)=\lambda_{2}^{\prime} F_{1}-v_{2}^{0}\left(\lambda_{1}^{\prime} F_{11}+\lambda_{2}^{\prime} F_{12}\right), \\
& L \delta^{\prime}(0)=\lambda_{2}^{\prime} F_{2}+v_{1}^{0}\left(\lambda_{1}^{\prime} F_{11}+\lambda_{2}^{\prime} F_{12}\right),
\end{aligned}
$$

where $L, F_{i}, F_{i j}$ are evaluated at $\left(v_{1}^{0}, v_{2}^{0}\right)$, and $\lambda_{i}$ at $s=0$. By (2.7) and (2.9) we have for the same evaluations

(2.16) $\lambda_{1}^{\prime} F_{1}+\lambda_{2}^{\prime} F_{2}=0, v_{1}^{0}\left(\lambda_{1}^{\prime} F_{11}+\lambda_{2}^{\prime} F_{12}\right)+v_{2}^{0}\left(\lambda_{1}^{\prime} F_{12}+\lambda_{2}^{\prime} F_{22}\right)=0$.

Hence,

$$
\begin{aligned}
\alpha^{\prime}(0)+\delta^{\prime}(0) & =0, \\
v_{1}^{0} \alpha^{\prime}(0)+v_{2}^{0} \beta^{\prime}(0) & =\lambda_{1}^{\prime}(0), \\
v_{1}^{0} \gamma^{\prime}(0)+v_{2}^{0} \delta^{\prime}(0) & =\lambda_{2}^{\prime}(0) .
\end{aligned}
$$

Now $0<\left(\lambda_{1}^{\prime}(0)\right)^{2}+\left(\lambda_{2}^{\prime}(0)\right)^{2}$. Placing $a_{0}=-\gamma^{\prime}(0), b_{0}=\alpha^{\prime}(0)=-\delta^{\prime}(0)$, $c_{0}=\beta^{\prime}(0)$, we conclude that $a_{0}, b_{0}, c_{0}$ are not all zero, and, using (2.15), that (2.6) holds at $\left(v_{1}, v_{2}\right)$. The only restriction on $\left(v_{1}, v_{2}\right)$ was that $v_{1}^{0} v_{2}-v_{2}^{0} v_{1} \neq 0$. Hence, by continuity, (2.6) holds in $V_{0}$.

We prove now that constants $a, b, c$, not all zero, can be chosen so that

(2.18) $\left(b u_{1}+c u_{2}\right) F_{1}\left(u_{1}, u_{2}\right)-\left(a u_{1}+b u_{2}\right) F_{2}\left(u_{1}, u_{2}\right)=0, \quad\left(u_{1}, u_{2}\right) \in V$.

By our preceding analysis, if $P \in V$ and $V_{P}$ is an open disk in $V$ having center at $P$, there corresponds to $P$ and $V_{P}$ a set of constants $a_{P}, b_{P}$, 
$c_{P}$, not all zero, such that (2.18) holds in $V_{P}$ with $a_{P}, b_{P}, c_{P}$ in place of $a, b, c$. We observe that since the gradient of $F$ is nonvanishing in $V$, if (2.18) holds with two different sets of constants on a domain $D \subset V$, these constants must be proportional. Hence, if we normalize the $a_{P}, b_{P}, c_{P}$, so that the first one which is not zero is one, then $a_{P}$, $b_{P}, c_{P}$ are uniquely determined. We show that the normalized $a_{P}, b_{P}$, $c_{P}$ are independent of $P$. Let $P_{0}:\left(v_{1}^{0}, v_{2}^{0}\right)$ be a fixed point in $V$, and let $P_{1}:\left(v_{1}^{1}, v_{2}^{1}\right)$ be an arbitrary second point. Let $\Gamma: u_{1}=\chi_{1}(t), u_{2}=\chi_{2}(t)$, $0 \leqq t \leqq 1, \chi_{i}(0)=v_{i}^{0}, \chi_{i}(1)=v_{i}^{1}$, be a Jordan arc in $V$ with endpoints at $P_{0}, P_{1}$. Let $E$ be the set of points $t$ on $[0,1]$ such that $a_{P}=a_{P_{0}}, b_{P}=b_{P_{0}}$, $c_{P}=c_{P_{0}}$, where $P$ has coordinates $\left(\chi_{1}(t), \chi_{2}(t)\right) . E$ is not void, and has on $[0,1]$ a least upper bound $t^{\prime}$, say. Utilizing our remark on the proportionality of constants for a domain $D$, we find first that $t^{\prime} \in E$, and secondly that $t^{\prime}=1$. Thus $a_{P_{1}}=a_{P_{0}}, b_{P_{1}}=b_{P_{0}}, c_{P_{1}}=c_{P_{0}}$. Taking $a=a_{P_{0}}, b=b_{P_{0}}, c=c_{P_{0}}$, our conclusion relative to (2.18) follows.

The balance of the proof rests on (2.4) and (2.18). Let $a, b, c$, be constants, not all zero, for which (2.18) holds. Write $\omega\left(u_{1}, u_{2}\right)=a u_{1}^{2}$ $+2 b u_{1} u_{2}+c u_{2}^{2}$. From (2.4) and (2.18) we obtain

$$
\omega F_{1}=\left(a u_{1}+b u_{2}\right) \xi(F), \quad \omega F_{2}=\left(b u_{1}+c u_{2}\right) \xi(F)
$$

for $\left(u_{1}, u_{2}\right) \in V$. We show first that $\omega \neq 0$ on $V$. Suppose, if possible, that $\left(v_{1}, v_{2}\right) \in V$ and $\omega\left(v_{1}, v_{2}\right)=0$. Since $\xi(F) \neq 0$, it then follows that $a v_{1}+b v_{2}=0, b v_{1}+c v_{2}=0$. Since $V$ does not contain the origin, we then have $b^{2}-a c=0$. Not both $a$ and $c$ vanish, and we can assume without loss of generality that $a \neq 0$. In this case we have $\omega=\left(a u_{1}+b u_{2}\right)^{2} / a$ in $V$, and accordingly, by (2.19), $\left(a u_{1}+b u_{2}\right)^{2} F_{1}=a\left(a u_{1}+b u_{2}\right) \xi(F)$ in $V$. From this relation we obtain $\left(a u_{1}+b u_{2}\right) F_{1}=a \xi(F)$ in $V$ except possibly on the line $a u_{1}+b u_{2}=0$. By continuity we then have $\xi(F)$ $=\left(a v_{1}+b v_{2}\right) F_{1} / a=0$ at $\left(v_{1}, v_{2}\right)$, which is a contradiction. Thus, $\omega \neq 0$ in $V$.

From (2.19) we have

$$
d F=(1 / 2) \xi(F) \omega^{-1} d \omega, \quad\left(u_{1}, u_{2}\right) \in V .
$$

Let $\eta$ be arbitrary on $(m, M)$. Motivated by (2.20) we define $\phi$ by (2.3). Then $\phi \in C^{\prime \prime}$ and $\phi^{\prime} \neq 0$ on $(m, M)$. From (2.20) we get

$$
\phi^{-1}(F) d \phi(F)=(1 / 2) \omega^{-1} d \omega, \quad\left(u_{1}, u_{2}\right) \in V .
$$

Since $\omega \neq 0$, we can normalize $a, b, c$ so that at an arbitrary point $\left(v_{1}, v_{2}\right)$ in $V$ we have $0<\omega$ and $\phi\left(F\left(v_{1}, v_{2}\right)\right)=\omega^{1 / 2}\left(v_{1}, v_{2}\right)$. The $\omega$ of our theorem is then determined. From (2.21) we obtain $\phi(F)=\omega^{1 / 2}$ in $V$. But $\phi$ has an inverse $\Phi$. Thus, $F=\Phi\left(\omega^{1 / 2}\right)$ in $V$. This completes the proof. 
3. Solutions of (1.2). We now consider briefly the integration of (1.2) when $f$ has the form (1.3) and $Z, A, C$ satisfy (1.4). We assume that $A \in C^{0}, Z \in C^{\prime}, C \in C^{\prime}$, and that the equations (1.4) hold on an interval $m<y<M$. We note that the first equation in (1.4) implies that $Z$ vanishes at most once on $(m, M)$. Also, the two equations imply that $Y=Z^{3} C$ satisfies $Y^{\prime}=4 A Y$. Hence, $Z C \neq 0$ or $Z C \equiv 0$ on $(m, M)$. It suffices then to treat two cases: Case I. $Z \neq 0$ on $(m, M)$; Case II. $C \equiv 0$ and $Z=0$ at some one point of $(m, M)$.

Case $\mathrm{I}$. For the case $Z \neq 0$, Theorem 2 is directly applicable. For $f$ in the form (1.3), $f(y, 0,0,1) \equiv Z(y)$. We then define $\phi$ by (2.3) with $\xi=Z$ and $\eta$ arbitrary on $(m, M)$. We may observe that because of (1.4) $\phi$ can be written

$$
\phi(y)=Z^{-1}(\eta) Z(y) \exp \left(-\int_{\eta}^{y} A(t) d t\right), \quad m<y<M .
$$

Denoting by $\Phi$ the inverse of $\phi$, we have $\Phi(t) \in C^{\prime \prime}, \Phi \neq 0$ on $g<t<G$, where $(g, G)$ is the range of $\phi$. Plainly, $0 \leqq g$. It remains to determine $\omega$. Let $\omega\left(u_{1}, u_{2}\right)=a u_{1}^{2}+2 b u_{1} u_{2}+c u_{2}^{2}$ be an arbitrary homogeneous polynomial of degree 2 which is positive at some point in the plane. Put $\Delta=b^{2}-a c$, and let $V$ be the (or a) domain determined by $0<\omega$, $g<\omega^{1 / 2}<G$. Suppose that $w(x), q(x)$ are arbitrary functions satisfying $w \in C^{\prime}, w \neq 0, q \in C^{0}$ on an interval $J$. Let $x_{0}$ be a point of $J$ and let $y_{0}, y_{0}^{\prime}$ be arbitrary initial values, where $m<y_{0}<M$. Evidently we can find $\left(v_{1}, v_{2}\right)$ in $V$ such that $\omega_{0}^{1 / 2} \equiv \omega^{1 / 2}\left(v_{1}, v_{2}\right)=\phi\left(y_{0}\right)$. Furthermore, $v_{1}^{\prime}, v_{2}^{\prime}$ can be determined so that $v_{1} v_{2}^{\prime}-v_{2} v_{1}^{\prime}=w\left(x_{0}\right), \Phi\left(\omega_{0}^{1 / 2}\right)\left[v_{i}, v_{i}^{\prime}\right] \omega_{0}^{-1 / 2}$ $=y_{0}^{\prime}$, where $\left[v_{i}, v_{i}^{\prime}\right]=a v_{1} v_{1}^{\prime}+b v_{1} v_{2}^{\prime}+b v_{1}^{\prime} v_{2}+c v_{2} v_{2}^{\prime}$. Denote by $u_{1}(x)$, $u_{2}(x)$ the solutions of (1.1) on $J$ with initial values $u_{i}\left(x_{0}\right)=v_{i}, u_{i}^{\prime}\left(x_{0}\right)$ $=v_{i}^{\prime}$. Then $u_{1}(x), u_{2}(x)$ have Wronskian $w$ and $\left(u_{1}, u_{2}\right) \in V$ for $x$ in an interval $I$ containing $x_{0}$. Defining $y$ by $(1 / 2) \ln \omega=\int_{\eta}^{y} Z^{-1}(t) d t$, and making use of (1.1), the first equation in (1.4) and the identity

$$
\left[u_{i}, u_{i}\right]\left[u_{i}^{\prime}, u_{i}^{\prime}\right]=\left[u_{i}, u_{i}^{\prime}\right]^{2}-\Delta\left(u_{1} u_{2}^{\prime}-u_{2} u_{1}^{\prime}\right)^{2},
$$

we find that $y=y(x)=\Phi\left(\omega^{1 / 2}\left(u_{1}(x), u_{2}(x)\right)\right)$ satisfies

$$
y^{\prime \prime}=w^{\prime}(x) w^{-1}(x) y^{\prime}+q(x) Z(y)+A(y)\left(y^{\prime}\right)^{2}-\Delta Z(y) \omega^{-2} w^{2}(x) .
$$

But

$$
C(y)=C(\eta) Z^{3}(\eta) Z^{-3}(y) \exp \left(4 \int_{\eta}^{y} A(t) d t\right)=C(\eta) Z^{-1}(\eta) Z(y) \omega^{-2}
$$

Hence, $y$ satisfies (1.2) on $I$ if $\Delta=-C(\eta) Z^{-1}(\eta)$. We have, further, $y\left(x_{0}\right)=\Phi\left(\omega_{0}^{1 / 2}\right)=y_{0}, \quad y^{\prime}\left(x_{0}\right)=\Phi^{\prime}\left(\omega_{0}^{1 / 2}\right)\left[v_{i}, v_{i}^{\prime}\right] \omega_{0}^{-1 / 2}=y_{0}^{\prime} . \quad$ Accordingly, 
for Case I, if $\Delta=-C(\eta) Z^{-1}(\eta)$, then for arbitrary $w(x), q(x)$ having the properties prescribed above, $y=\Phi\left(\omega^{1 / 2}\left(u_{1}, u_{2}\right)\right)$, together with solutions $u_{1}(x), u_{2}(x)$ of (1.1) having Wronskian $w$, and such that $\left(u_{1}(x), u_{2}(x)\right) \in V$ provide the general solution of (1.2).

Case II. If $Z$ vanishes at a point of $(m, M)$, then the definition (2.3) is inappropriate. However, if $\eta$ is chosen so that $Z(\eta) \neq 0$, then (3.1) is applicable. Defining $\phi$ by (3.1), and using the first equation in (1.4), we find that

$$
\phi^{\prime}(y)=Z^{-1}(\eta) \exp \left(-\int_{\eta}^{y} A(t) d t\right), \quad m<y<M .
$$

Thus, $\phi \in C^{\prime \prime}$ and $\phi^{\prime} \neq 0$ on $(m, M)$. Denoting by $\Phi$ the inverse of $\phi$, we have again $\Phi(t) \in C^{\prime \prime}, \Phi^{\prime} \neq 0$ on $g<t<G$, where $(g, G)$ is the range of $\phi$. In this case, $g<0<G$. Let $\tilde{\omega}\left(u_{1}, u_{2}\right)=a u_{1}+b u_{2}$ be an arbitrary nontrivial linear function. Determine $V$ by $g<\tilde{\omega}<G$. Introducing $w, q, u_{1}, u_{2}$ as in the preceding paragraph, we find that $y=\Phi\left(a u_{1}+b u_{2}\right)$ satisfies

$$
y^{\prime \prime}=w^{\prime} w^{-1} y^{\prime}+q Z(y)+A(y)\left(y^{\prime}\right)^{2} .
$$

Since in the case under consideration we have $C \equiv 0$, we see that $y=\Phi\left(\tilde{\omega}\left(u_{1}, u_{2}\right)\right)$, together with appropriate solutions of (1.1), provide the general solution (1.2) for Case II.

We may observe that canonical forms for the solution of (1.2) are

$$
y=\Phi\left(\left[u_{1}^{2}+u_{2}^{2}\right]^{1 / 2}\right), \quad y=\Phi\left(\left[u_{1}^{2}-u_{2}^{2}\right]^{1 / 2}\right), \quad y=\Phi\left(u_{1}\right),
$$

where $u_{1}, u_{2}$ are solutions of (1.1).

\section{REFERENCES}

1. R. T. Herbst, The equivalence of linear and nonlinear differential equations, Proc. Amer. Math. Soc. 7 (1956), 95-97.

2. E. Pinney, The nonlinear differential equation $y+p(x) y+c y^{-3}=0$, Proc. Amer. Math. Soc. 1 (1950), 681.

3. J. M. Thomas, Equations equivalent to a linear differential equation. Proc. Amer. Math. Soc. 3 (1952), 899-903.

University of Glasgow, Glasgow, Scotland and DUKE UNIVERSITY 